\title{
Temperature and Pressure Dependence of the Linewidth for an Internal Mode in the Solid Phases of Benzene
}

\author{
H. Yurtseven* And M.G. ŞENOL \\ Department of Physics, Middle East Technical University, 06531 Ankara, Turkey
}

(Received November 20, 2012; in final form July 15, 2013)

\begin{abstract}
The pressure dependence of the Raman linewidths for the $\nu_{1}$ breathing mode is analyzed using the experimental data from the literature for the solid phases (I, II, III and III') in benzene. Increase (I, III and III') and decrease (II) in the Raman linewidths are described by a power-law analysis with the critical exponent $\beta$. The $\beta$ values we find, which characterize the phase transition are in favour of the first order among those phases considered in benzene. The temperature and pressure dependence of the frequencies and linewidths of various Raman modes can be analyzed using a power-law formula, as studied here for the solid phases (I, II, III and III') in benzene when the experimental data are available in the literature.
\end{abstract}

DOI: 10.12693/APhysPolA.124.698

PACS: 64.60.-i, 05.70.Fh

\section{Introduction}

Benzene is an organic compound with the formula $\mathrm{C}_{6} \mathrm{H}_{6}$ that has many applications in technology. It has various solid phases, namely, I, II, III, III' and IV. At very low pressures and high temperatures it melts. At higher pressures and temperatures, polymer phases (polymer 1 and polymer 2) occur. It decomposes at above $600{ }^{\circ} \mathrm{C}$ up to about $10 \mathrm{GPa}$, as shown in the experimental [1] $T-P$ phase diagram (Fig. 1).

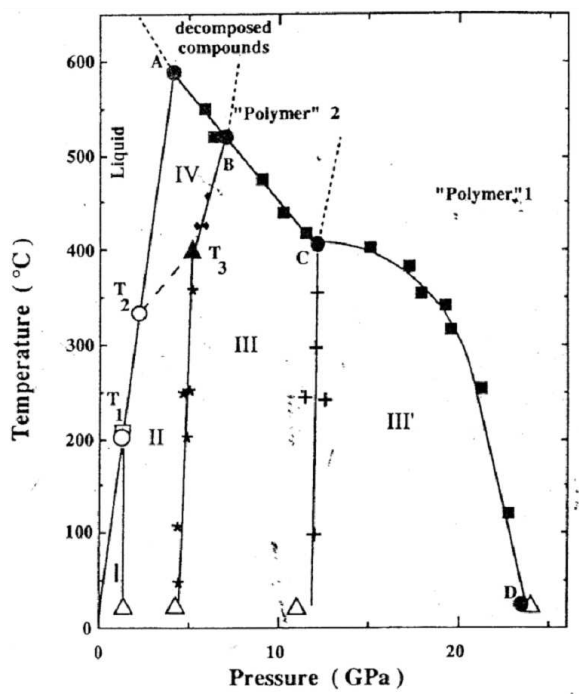

Fig. 1. Experimental phase diagram of benzene [1].

Previously, the $T-P$ phase diagrams of benzene have been obtained using different experimental techniques [2-6]. In this phase diagram (Fig. 1), $T_{1}, T_{2}$, and $T_{3}$

\footnotetext{
*corresponding author; e-mail: hamit@metu.edu.tr
}

are triple points so that the transitions of liquid-I, II, and II-III are of a first order. It has been suggested [6] that III-III' transition is of a second order and III'-IV transition is also of the first order, where the solid IV is a proposed phase that has been obtained by the Raman scattering up to $14 \mathrm{GPa}$ at room temperature $[7,8]$. Recently, we have calculated the $T-P$ phase diagram of benzene on the basis of the experimental diagram [1] as given in Fig. 1 using the mean field theory $[9,10]$.

Various physical properties of benzene have been studied experimentally and theoretically, as reported in the literature. Near the phase transitions, the thermodynamic quantities such as specific heat, thermal expansion and isothermal compressibility, and the spectroscopic parameters (frequency, intensity, and bandwidth) have been measured or they have been calculated as functions of temperature and pressure. Near the melting point, its molar volume [11] and the thermal expansion [12] have been measured. We have also studied molar volume of benzene near the melting point as functions of temperature [13] and pressure [14]. Very recently, we have studied the Pippard relations close to the melting point in this molecular solid [15].

Spectroscopic techniques such as Raman $[6,8,16,17]$, infrared [17-20] and X-rays [19, 20] have been used to study the phase transformations in benzene. Recently, we have investigated [21] vibrational frequencies at various pressures in the solid phase II of benzene using the experimental volume [6] and Raman [20] data.

In this study, we analyze the pressure dependence of the Raman linewidths of the internal mode $\left(\nu_{1}\right)$, which were measured experimentally [1] for the solid phases of I, II, III and III' $\left(T=103^{\circ} \mathrm{C}\right)$ in benzene. We also analyze the pressure dependence of the Raman linewidths of this mode for the phases of II, III and III' $\left(T=360^{\circ} \mathrm{C}\right)$. We then calculate the temperature dependence of the linewidths of the $\nu_{1}$ mode in the solid phases studied in benzene. 


\section{Calculations and results}

The pressure dependence of the linewidth can be analyzed close to the phase transitions in benzene according to a power-law relation

$$
\Gamma=A\left(\frac{P}{P_{\mathrm{c}}-P}\right)^{2 \beta},
$$

where $\beta$ is the critical exponent for the order parameter and $A$ is the amplitude. This relation describes the divergence behavior of the linewidth close to the critical pressure $P_{\mathrm{c}}$. In this study, we analyzed the observed data [1] for the Raman linewidth of the internal mode $\left(\nu_{1}\right)$ at various pressures for the solid phases of I, II, III and III' $^{\prime}$ in benzene according to Eq. (1). From our analysis of the experimental data [1], values of the critical exponent $\beta$ and the amplitude $A$ were extracted for the phases of I, II, III and III' $\left(T=103^{\circ}\right)$ and II, III and III' $\left(T=360^{\circ}\right)$, as given in Tables I and II, respectively. The observed data [1] plotted for the linewidth of the $\nu_{1}$ mode against pressure are given in Figs. 2 and 3 for constant temperatures of 103 and $360^{\circ} \mathrm{C}$, respectively.

TABLE I

Values of the critical exponent $\beta$ and the amplitude $A$, which were extracted from our analysis using the experimental data [1] according to Eq. (1) within the pressure ranges at $T=103{ }^{\circ} \mathrm{C}$ for the solid phases indicated in benzene. Values of $P_{\mathrm{c}}$ are also given.

\begin{tabular}{c|c|c|c|c}
\hline \hline Phases & $\beta$ & $A\left[\mathrm{~cm}^{-1}\right]$ & $P_{\mathrm{c}}[\mathrm{GPa}]$ & $\begin{array}{c}\text { Pressure range } \\
{[\mathrm{GPa}]}\end{array}$ \\
\hline I & 0.06 & 3.40 & 1.6 & $0.7<P<1.3$ \\
II & -0.03 & 3.56 & 4.5 & $1.6<P<4.1$ \\
III & 0.03 & 3.32 & 11.9 & $4.7<P<11.5$ \\
III' $^{\prime}$ & -0.10 & 7.16 & 11.9 & $12.8<P<19.3$
\end{tabular}

TABLE II

As Table I, for $T=360^{\circ} \mathrm{C}$.

\begin{tabular}{c|c|c|c|c}
\hline \hline Phases & $\beta$ & $A\left[\mathrm{~cm}^{-1}\right]$ & $P_{\mathrm{c}}[\mathrm{GPa}]$ & $\begin{array}{c}\text { Pressure range } \\
{[\mathrm{GPa}]}\end{array}$ \\
\hline II & -0.03 & 6.03 & 5.2 & $2.5<P<4.9$ \\
III & 0.03 & 5.22 & 11.8 & $5.1<P<11.3$ \\
III $^{\prime}$ & -0.04 & 8.20 & 11.8 & $12.1<P<17.3$
\end{tabular}

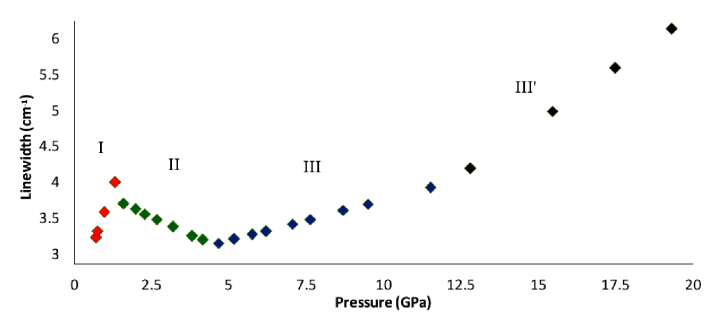

Fig. 2. The observed linewidth [1] of the $\nu_{1}$ mode as a function of pressure for the solid phases of I, II, III and III' of benzene at $T=103{ }^{\circ} \mathrm{C}$.

The temperature dependence of the Raman linewidths for the $\nu_{1}$ mode can be predicted using the observed data for the Raman frequencies at various pressures for the

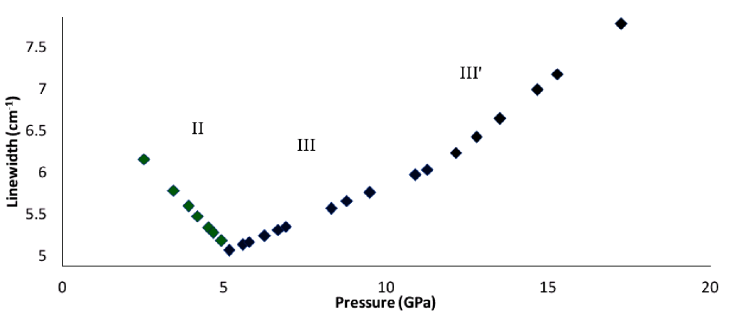

Fig. 3. The observed linewidth [1] of the $\nu_{1}$ mode as a function of pressure for the solid phases of II, III and III' of benzene at $T=360^{\circ} \mathrm{C}$

phases II, III and III' in benzene. For this calculation, we first obtained the values of the slope $\mathrm{d} P / \mathrm{d} T$ along the I-II (up to $T_{1}$ ), II-III (up to $T_{3}$ ) and III-III' (up to $C$ ) from the $T-P$ phase diagram of benzene [1], as given in Fig. 1.

Using the approximate relation

$$
\frac{\mathrm{d} T}{\mathrm{~d} P}=\frac{T-T_{\mathrm{c}}}{P-P_{\mathrm{c}}}
$$

along the phase line between the solid phases of benzene, through Eq. (1), the temperature dependence of the linewidth $\Gamma$ can be expressed as

$$
\Gamma=A\left[\frac{P_{\mathrm{c}}}{T_{\mathrm{c}}-T}\left(\frac{\mathrm{d} T}{\mathrm{~d} P}\right)\right]^{2 \beta} .
$$

Thus, using the slope values of $\mathrm{d} P / \mathrm{d} T$ which were obtained from the $T-P$ phase diagram (Fig. 1), and the experimental data [1] for the linewidth $\Gamma$ at various pressures, values of the linewidth $\Gamma$ at constant temperatures were calculated by Eq. (3), as given in Table III $\left(T=103^{\circ} \mathrm{C}\right)$ and Table IV $\left(T=360^{\circ} \mathrm{C}\right)$.

TABLE III

Values of the critical exponent $\beta$ and the amplitude $A$ (Eq. (1)), the critical pressure $P_{\mathrm{c}}$, the slope $\mathrm{d} P / \mathrm{d} T$ and the values of the linewidth $(\Gamma)$ at constant $T_{\mathrm{c}}-T$ (Eq. (3)) for the phase lines indicated $\left(T=103{ }^{\circ} \mathrm{C}\right)$ in benzene.

\begin{tabular}{c|c|c|c|c|c|c}
\hline \hline Phases & $\beta$ & $\begin{array}{c}\mathrm{A} \\
{\left[\mathrm{cm}^{-1}\right]}\end{array}$ & $\begin{array}{c}P_{\mathrm{c}} \\
{[\mathrm{GPa}]}\end{array}$ & $\begin{array}{c}\left(T_{\mathrm{C}}-T\right) \\
\times 10^{-4} \\
{\left[{ }^{\circ} \mathrm{C}\right]}\end{array}$ & $\begin{array}{c}\mathrm{d} P / \mathrm{d} T \\
\times 10^{3} \\
{\left[\mathrm{GPa} /{ }^{\circ} \mathrm{C}\right]}\end{array}$ & $\begin{array}{c}\text { Linewidth } \\
{\left[\mathrm{cm}^{-1}\right]}\end{array}$ \\
\hline I-II & 0.04 & 3.47 & 1.6 & 1.53 & 4.46 & 3.77 \\
II-III & 0.03 & 3.43 & 4.5 & 42.9 & 0.44 & 3.41 \\
III-III' & 0.07 & 5.23 & 11.8 & 75.8 & 0.64 & 3.50
\end{tabular}

TABLE IV

As Table III, for $T=360^{\circ} \mathrm{C}$.

\begin{tabular}{c|c|c|c|c|c|c}
\hline \hline Phases & $\beta$ & $\begin{array}{c}A \\
{\left[\mathrm{~cm}^{-1}\right]}\end{array}$ & $\begin{array}{c}P_{\mathrm{c}} \\
{[\mathrm{GPa}]}\end{array}$ & $\begin{array}{c}\left(T_{\mathrm{C}}-T\right) \\
\times 10^{-3} \\
{\left[{ }^{\circ} \mathrm{C}\right]}\end{array}$ & $\begin{array}{c}\mathrm{d} P / \mathrm{d} T \\
\times 10^{3} \\
{\left[\mathrm{GPa} /{ }^{\circ} \mathrm{C}\right]}\end{array}$ & $\begin{array}{c}\text { Linewidth } \\
{\left[\mathrm{cm}^{-1}\right]}\end{array}$ \\
\hline II-III & 0.03 & 5.62 & 5.2 & 1.9 & 0.53 & 5.48 \\
III-III $^{\prime}$ & 0.03 & 6.71 & 11.8 & 3.2 & 1.09 & 5.58
\end{tabular}

Values of the critical exponent $\beta$ and the amplitude $A$, which we extracted from Eq. (1), with the $P_{\mathrm{c}}$ values are also given in these tables.

We plot in Fig. 4 the Raman linewidth $\left(\nu_{1}\right)$ calculated (Eq. (3)) as a function of temperature for the phases of I, 


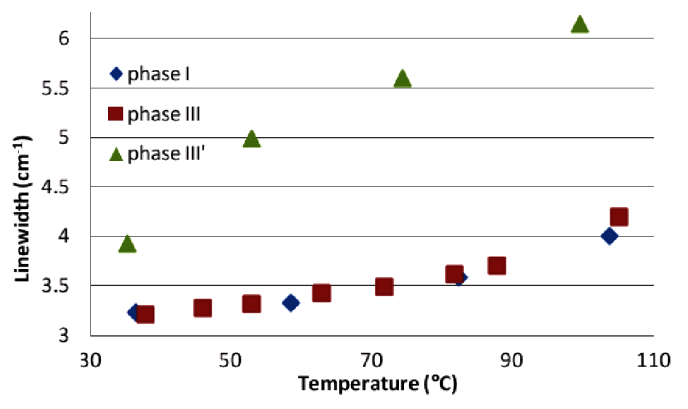

Fig. 4. The Raman linewidth of the $\nu_{1}$ mode as a function of temperature for the solid phases of I, III and III', which was calculated by Eq. (3) using the $\Gamma$ vs. $P$ data at $103{ }^{\circ} \mathrm{C}$ in benzene.

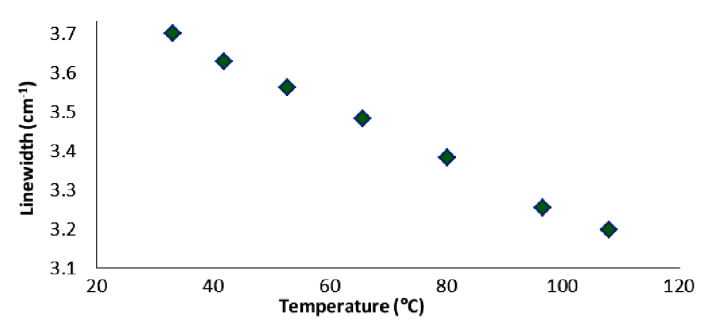

Fig. 5. The Raman linewidth of the $\nu_{1}$ mode as a function of temperature for the solid phase of II, which was calculated by Eq. (3) using the $\Gamma$ vs. $P$ data at $103^{\circ} \mathrm{C}$ in benzene.

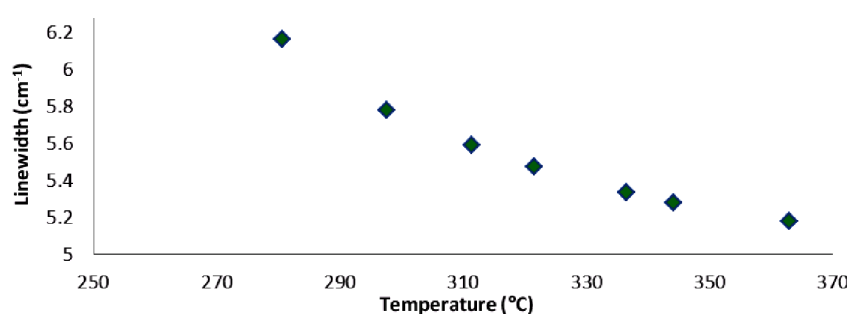

Fig. 6. The Raman linewidth of the $\nu_{1}$ mode as a function of temperature for the solid phases of II, which was calculated by Eq. (3) using the $\Gamma$ vs. $P$ data at $360^{\circ} \mathrm{C}$ in benzene.

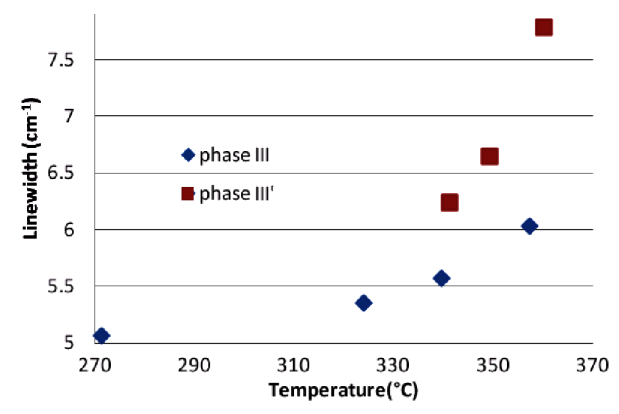

Fig. 7. The Raman linewidth of the $\nu_{1}$ mode as a function of temperature for the solid phases of III and III', which was calculated by Eq. (3) using the $\Gamma$ vs. $P$ data at $360{ }^{\circ} \mathrm{C}$ in benzene.
III and III', using the $\Gamma$ vs. $P$ data at $103^{\circ} \mathrm{C}$ in benzene. Figure 5 gives our $\Gamma$ plot vs. $T$ for the phase II using the $\Gamma$ vs. $P$ data at $103^{\circ} \mathrm{C}$.

Similarly, we plot in Fig. 6 the Raman linewidth $\left(\nu_{1}\right)$ calculated (Eq. (3)) as a function of temperature for the phase II using the $\Gamma$ vs. $P$ data at $360^{\circ} \mathrm{C}$.

Our plot of $\Gamma$ vs. $T$ for the phases III and III $^{\prime}$ is given in Fig. 7 using the $\Gamma$ vs. $P$ data at $360^{\circ} \mathrm{C}$.

\section{Discussion}

The analysis of the experimental linewidths $\Gamma$ [1] for the breathing mode $\left(\nu_{1}\right)$ was performed have as functions of pressure at constant temperatures of $103^{\circ} \mathrm{C}$ (phases I, II, III and III' $^{\prime}$ ) and $360^{\circ} \mathrm{C}$ (phases II, III and III' $^{\prime}$ in benzene. In order to investigate the phase transitions between the solid phases considered in benzene, a power-law analysis (Eq. (1)) was employed and by determining the values of the critical exponent $\beta$ (Eq. (1)), the phase transition (first order or second order) was characterized in this molecular crystal. Previously, the phase boundaries between the solid phases were determined by the changes in the slopes of the linewidth against pressure and also the minimum in the linewidth [1]. This has been criticized in the evolution of the linewidth $(\Gamma)$ of the breathing mode $\left(\nu_{1}\right)$ with pressure, since other effects could be detected in the pressure shift of this mode or other modes of benzene [20]. It has been argued [22] that the linewidth-pressure shift or the lifetime of the mode reflects the entire crystal dynamics. So that the anomalous behavior of the linewidth with the pressure can be attributed to the coupling with the other phonons [20]. It has also been argued [22] that a cusp minimum in the linewidth does not indicate the phase boundaries in benzene, which can be explained as the blueshift of the one density of states (DOS) with increasing pressure on the basis of the observed symmetric stretching mode $\left(\nu_{1}\right)$ of the $\mathrm{NO}_{3}^{-}$ion in $\mathrm{NaNO}_{3}$ and $\mathrm{KNO}_{3}$ crystals within the same crystal phase [23]. In fact, this has been observed in the solid phases of I, II, III and III' of benzene from the $\nu-P$ plots of the various external and internal modes whose Raman frequencies shift to blue with increasing pressure [6].

On the basis of the values of the critical exponent $\beta$ which describes the anomalous behavior of the linewidth $\Gamma$, the mechanism of the transition among the solid phases of I, II, III and III' can be explained. Our value of $\beta=0.04$ at $103^{\circ} \mathrm{C}$ (Table III) indicates a first order transition between the solid phases I and II, as first reported by Bridgman [2]. Also, our values of $\beta=0.03$ at $103{ }^{\circ} \mathrm{C}$ (Table III) and $360^{\circ} \mathrm{C}$ (Table IV) indicate a first order transition between the solid phases II and III, which has been found previously at nearly $4 \mathrm{GPa}[6,17]$. On the basis of our values of $\beta=0.07$ at $103{ }^{\circ} \mathrm{C}$ (Table III) and $\beta=0.03$ at $360^{\circ} \mathrm{C}$ (Table IV), the III-III' transition can also be considered as a first order. This is not in agreement with the second order transition from the phase III to III', as obtained experimentally [1]. However, this 
transition changes from a first order to the second order at $11 \mathrm{GPa}$, as pointed out previously [6].

As observed experimentally [1], the linewidth $\Gamma$ increases with the pressure in the solid phases of I, III and $\mathrm{III}^{\prime}\left(103^{\circ} \mathrm{C}\right)$ and, in the solid phases of III and III $^{\prime}$ $\left(360^{\circ} \mathrm{C}\right)$, whereas it decreases with increasing pressure in phase II (103 and $\left.360^{\circ} \mathrm{C}\right)$. When pressure increases in the phases of I, III and III $^{\prime}\left(103^{\circ} \mathrm{C}\right)$ and, in the phases of III and $\mathrm{III}^{\prime}\left(360^{\circ} \mathrm{C}\right)$ the $\nu_{1}$ line shifts toward the higher frequencies and it broadens as observed experimentally [1]. In phase II, the frequency shifts of the Raman bands with the pressure are smaller than those in phase I [6]. There is an increasing ordering in the solid with increasing pressure, which causes a decreasing entropy and a decreasing linewidth in phase II [1] whereas in the solid phases of I, III and III' the entropy increases (disorder entropy) accompanied with increasing linewidth. By increasing pressure, phase I with the orthorhombic structure is translated into the phase II that has the lower symmetry of the monoclinic structure with the reduction of the number of molecules per unit cell. This results in narrowing the linewidth with larger splitting [6] in phase II. However, it has been pointed out [20] that this narrowing of the $\nu_{1}$ mode as observed during the compression of the sample in phase II [1] has not been confirmed by the time-resolved stimulated Raman scattering in the same pressure range using the time-resolved stimulated Raman scattering, which given broadening linewidth of this mode [24]. This line narrowing of the $\nu_{1}$ mode also occurs as we calculated here at various temperatures in phase II on the basis of the observed $\Gamma$ vs. $P$ data [1]. It has been suggested $[25,26]$ that the relaxation dynamics of the $\nu_{1}$ vibrational mode was dominated by the $\nu_{10}$ mode at $\approx 860 \mathrm{~cm}^{-1}$. With increasing temperature, the linewidth of the $\nu_{1}$ mode narrows when coupled to the $\nu_{18}$ mode $\left(1037 \mathrm{~cm}^{-1}\right)$ which is driven by the lattice mode. Since the lattice mode has lower energy, this gives rise to the $\nu_{1}$ line narrowing during the compression, as pointed out previously [20]. Although in phase I when there is no pressure act $274 \mathrm{~K}$, the most intense line at $991 \mathrm{~cm}^{-1}$ is the $\nu_{1}\left(A_{\mathrm{g}}\right)$ ring breathing mode of benzene [6], the linewidths of the other Raman active modes of this molecular crystal can be studied as a function of pressure. By the same kind of analysis which we did here for the $\nu_{1}$ mode, variation of linewidth with the pressure can give some additional information about the mechanism of the phase transitions in benzene.

\section{Conclusions}

The observed Raman linewidths of the internal mode $\nu_{1}$ mode were analyzed by a power-law formula for the solid phases of benzene. Our values of the critical exponent from the pressure dependence of the linewidths indicate that the transitions among the solid phases of I, II and III (III') are of a first order.

The pressure dependence of the linewidth can be studied experimentally for some other modes in benzene. The temperature dependence of the linewidths of various Raman modes can also be studied experimentally to ex- amine our predictions given here. Thus, by means of the power-law analysis the phase transition between the solid phases can be characterized in benzene.

\section{References}

[1] F. Cansell, D. Fabre, J.P. Petitet, J. Chem. Phys. 99, 7300 (1993).

[2] P.W. Bridgman, J. Chem. Phys. 9, 794 (1941).

[3] G.J. Piermarini, A.D. Mighell, C.E. Weir, S. Block, Science 165, 1250 (1969).

[4] S. Block, C.E. Weir, G.J. Piermarini, Science $\mathbf{1 6 9}$, 586 (1970).

[5] J. Akella, G.C. Kennedy, J. Chem. Phys. 55, 793 (1971).

[6] M.M. Thiery, J.M. Leger, J. Chem. Phys. 89, 4255 (1988).

[7] M.M. Thiery, I. Spain, K. Kobashi, Solid State Commun. 54, 95 (1985).

[8] M.M. Thiery, D. Fabre, I. Spain, K. Kobashi, Physica B 139-140, 520 (1986).

[9] S. Şen, H. Yurtseven, J. Optoelectron. Adv. Mater. Symp. 1, 517 (2009).

[10] S. Şen, H. Yurtseven, Int. J. Trans. Phenom. 12 319 (2011).

[11] P. Figuiere, A.H. Fuchs, M. Ghelfenstein, H. Szwarc, J. Phys. Chem. Solids 39, 19 (1978).

[12] Ph. Pruzan, D.H. Liebenberg, R.L. Mills, J. Phys. Chem. Solids 47, 949 (1986).

[13] H. Yurtseven, T. Unsal, High Temp. Mater. Proc. 26, 365 (2007).

[14] H. Yurtseven, T. Unsal, Tsinghua Sci. Tech. 12, 624 (2007).

[15] H. Yurtseven, A.S. Karakuş, Int. J. Pharmac., Chem. Bio. Sci. 2, 729 (2012).

[16] W.D. Ellenson, M. Nicol, J. Chem. Phys. 61, 1380 (1974).

[17] D.M. Adams, R. Applety, J. Chem. Soc. Faraday Trans. 273, 1896 (1977).

[18] A. Anderson, B. Piwowar, W. Smith, Spectrosc. Lett. 31, 1811 (1999).

[19] L. Ciabini, M. Santoro, R. Bini, V. Schettino, J. Chem. Phys. 115, 3742 (2001).

[20] L. Ciabini, F.A. Gorelli, M. Santoro, R. Bini, V. Schettino, M. Mezouar, Phys. Rev. B 72, 094108 (2005).

[21] H. Yurtseven, B. Raşitoğlu, E. Kilit, J. Mol. Struct. 993, 428 (2011).

[22] S. Califano, V. Schettino, Int. Rev. Phys. Chem. 7, 19 (1988).

[23] M. Jordan, A. Schuch, R. Righini, G.F. Signorini, H.J. Jodl, J. Chem. Phys. 101, 3436 (1994).

[24] M. Baggen, M. van Exter, A. Lagendijk, J. Chem. Phys. 86, 2423 (1986).

[25] R.G. della Valle, R. Righini, Chem. Phys. Lett. 148, 45 (1988).

[26] R. Torre, R. Righini, L. Angeloni, S. Califano, J. Chem. Phys. 93, 2967 (1990). 